\title{
Genotyping of Mycobacterium leprae in Myanmar and supposed transmission mode
}

\author{
Khin Saw Aye ${ }^{1)}$, Yin Thet Nu Oo ${ }^{1)}$, Kyaw Kyaw²), Aye Aye Win¹, \\ Masanori MATSUOKA ${ }^{3) *}$ \\ 1) Immunology Research Division, Department of Medical Research (Lower Myanmar), Yangon, Myanmar \\ 2) Central Special Skin Clinic, Yangon General Hospital, Yangon, Myanmar \\ 3) Leprosy Research Center, National Institute of Infectious Diseases, Tokyo, Japan
}

[Received: 7 May, 2012 / Accepted: 28 May, 2012]

Key words : Genotyping, Leprosy, Mycobacterium leprae, Transmission mode, TTC repeats

The polymorphism of TTC repeats in Mycobacterium leprae was examined using bacilli from slit skin samples of leprosy patients attending at Central Special Skin Clinic, Yangon General Hospital and nasal swabs of their contacts to elucidate the possible mode of leprosy transmission. It was found that bacilli with different TTC genotypes were distributed among same household contacts and also harbored bacilli in patients were different TTC genotype from that harbored on the nasal mucus of the healthy contacts. Genotypes of TTC repeats were found to differ between husband under treatment and his wife and also mother under treatment and her sons living in same house. This study revealed that TTC genotype of bacilli harbored by household contacts was different with the TTC genotype by index cases. These results indicate that the family members get transmission from outside the dwellings rather than from commonly supposed their MB index cases. There might have been some infectious sources to which the populace had been commonly exposed outside the dwellings.

\section{Introduction}

Leprosy is a chronic infectious disease caused by Mycobacterium leprae infection. It has long being

\footnotetext{
*Corresponding author :

Leprosy Research Center, National Institute of Infectious Diseases

4-2-1, Aoba-Cho, Higashimurayama, Tokyo, 189-0002, Japan

TEL : +81-423-91-8211 FAX : +81-423-94-9092

E-mail : matsuoka@nih.go.jp
}

believed that the source of infection is untreated multibacillary leprosy patients. It has also been predicted that multidrug therapy (MDT) with strong bactericidal antibiotics (such as rifampicin) would reduce the source of infection and consequently interrupt further transmission to others. However, the number of new cases has shown no substantial decline especially other than India. It is reported that about 200,000 to 30,000 new cases are continuously found in the world every year (1), which 
suggests that the transmission of leprosy bacilli still occurs, especially in countries of endemicity. Elucidation of the mode of transmission would be essential to reduce newly transmitted cases. The differentiation of strains of leprosy bacilli by genomic polymorphism might be of great value in efforts to understand the mode of transmission of the disease. The range of molecular techniques for epidemiological analysis has expanded in recent years, and there are now many genotypic methods that allow a high level of discrimination between bacterial strains (references 2, 3, 4). Shin et al. discovered a genomic divergence of $M$. leprae by the variation of TTC repeats (5) and subdivided 34 isolates into 15 subtypes. Genotyping according to the TTC repeats for fragments amplified by PCR seemed to be feasible for molecular epidemiological analysis of leprosy transmission. A previous study by Saeki et al. revealed that $M$. leprae existed on the surface of nasal cavities of residents in areas with high prevalence (6). Here, we report the distribution of different TTC genotypes of $M$. leprae among family members of each household and inconsistent genotypes obtained from patients and their family members in the same dwelling. The results strongly supported the previously proposed hypotheses (7) on the existence of an infectious source(s) other than that of patients living with family members.

\section{Materials and Methods}

\section{Samples from patients}

To clarify whether the TTC genotype in one patient varies or not, genotypes of the bacilli obtained from various lesions of one patient were compared. Slit-skin smear samples (SSS) from 45 lesions of 22 patients, two SSS from 21 patients and 3 SSS from one patient, were obtained at Central Special Skin Clinic (CSSC), Yangon General Hospital. Samples were collected in the same manner as is used for routine slit-skin smear testing for bacterial index examination. The sample on the disposable surgical blade was soaked in $70 \%$ ethanol and kept at room temperature until test.

\section{Samples from patients and their contacts (who} develop new case later) in the same dwelling

TTC genotypes of the bacilli from the lesion of four multi-family cases, multiple leprosy case among family member or living in the same dwellings, were compared. Skin slit samples were collected from at least two lesions of each patient. The genotype of each isolate was examined as described below.

Case 1: A MB case supposed to be a index case and his son developed the disease later.

Case 2: The same as Case 1 in another house.

Case 3: Supposed index MB case and after 10 months of MDT, his daughter developed the disease.

Case 4: MB case and after 9 months of MDT, his brother developed the disease

\section{Samples from household contacts}

TTC genotypes of the bacilli from nasal swab specimen of 92 household contacts (HC) in 18 dwellings with 22 patients diagnosed at CSSC were examined. $\mathrm{HC}$ were defined as persons sleeping during the night under the same roof. Nasal swabs were taken by introducing cotton tip swabs (sterilized $J C B$ MENTIP, Japan) 2-3cm into each nostril successively, and rubbing gently on the lateral and median sides of each cavity. Swabs were immediately chilled (kept in ice box) and transported to the Immunology Research Division, DMR (Lower Myanmar) and analyzed.

\section{Preparation of template DNA and sequencing analysis}

The sample obtained from slit skin was removed 
from the blade and collected as a pellet by centrifugation at $10,000 \mathrm{rpm}$ for $20 \mathrm{~min}$ in $70 \%$ ethanol and then washed with phosphate-buffered saline. The template was prepared by treatment with lysis buffer according to the method described by Klatser et al, (8), and then the TTC genotype was examined.

Templates from nasal swab materials and slitskin samples were prepared by treatment with lysis buffer at $60^{\circ} \mathrm{C}$ overnight as described elsewhere (8), TTC repeats regions were amplified by PCR with the primers indicated by Shin et al. (5). Copy numbers of TTC repeats were examined by the direct sequencing of the PCR products. Briefly, the regions flanking TTC repeats were amplified using a G mixture and a FailSafe PCR system (EPICENTRE, Madison, Wis. USA). DNA samples for sequencing were recovered with a MinElute gel extraction kit (QIAGEN GmbH, Hilden, Germany) after $1.2 \%$ Seakem agarosegel electrophoresis of PCR products. Samples were sequenced with a BigDye terminator cycle sequencing FS Ready Reaction kit Ver. 1.1 (Perkin-Elmer Applied Biosystems, Norwalk, Conn.) and an ABI Prism 310 genetic analyzer (Perkin-Elmer). The nucleotide sequences obtained were analyzed using DNASIS software (Hitachi Software Engineering, Yokohama, Japan).

\section{Ethical approval}

The study was approved by the Institutional Ethical Review Committee of Department of Medical Research (Lower Myanmar). Informed consent was obtained from all subjects. Bacillary samples of nasal swabs and slit-skin smears were collected after informed consent was obtained.

\section{Results}

Genotype of the bacilli from the nasal swab

\section{samples}

Of $92 \mathrm{HC}$ in 18 dwellings, there were 30 (33\%) $\mathrm{HC}$ individuals carried the bacilli on the surface of their nasal cavities. TTC genotype of the bacilli from nasal mucus of $\mathrm{HC}$ in 14 dwellings out of 18 dwellings was identical. Genotype of the bacilli obtained in 4 multi-family cases different among family members (Table 1). Residents in these houses harbored different TTC genotypes from each other; their TTC genotypes were 9, 11, 12, 13, 14, 15, 16, 17,21 , and 22 repeats. The TTC repeats of the bacilli from the skin lesion of new MB case consisted of 11 copies and the TTC repeats of the bacilli from his nasal cavities was 15 , on the other hand the bacilli from his family contacts, wife and son, showed 14 and 17 copies respectively. The TTC repeats genotype of the bacilli from $\mathrm{PB}$ patient showed 21 copies but bacilli from his household contact (HC) nasal mucus showed 15 copies. The TTC repeats of the bacilli from another new MB case consisted of 13 copies, but the bacilli from his family contacts, two daughters and a son, showed 13, 16 and 9 copies (Table.1). The frequency of each TTC genotype of the bacilli obtained from 45 skin lesion and 52 nasal samples from 22 patients and $\mathrm{HC}$ were shown in Table 2. The most predominant genotype was 16 copies of TTC repeats and the $2^{\text {nd }}$ dominate type was 14 copies of TTC repeats.

\section{Genotype of the bacilli in the lesions}

From all 22 patients, 45 samples of different lesions showed identical genotypes. The most dominant genotype has 16 copies of TTC repeats in these patients. The other genotypes (number 9 , $11,12,13,14,15,16,17,21$ and 22 copies of TTC repeats) were detected. The frequency of each TTC genotype observed in samples from lesions of the patients and the nasal cavities of the residents is shown in Table 2. 
Comparison of TTC genotypes among patients in a dwelling

The TTC genotypes of $M$. leprae of supposed index and secondary cases were compared. The genotypes of index case patients in two multi-family cases harbored the bacilli with 13 and 22, and their son (secondary case) showed 9 and 17 copies of TTC repeats respectively. In case 3 who was MB case harbored bacilli with 11 copies of TTC repeats, after 10 months of MDT his daughter developed as secondary case and harbored bacilli with 14 TTC repeats. Another case 4 of household cases of two brothers showed different TTC genotypes (15 and 16 TTC repeats) within the family (Table $3)$.

\section{Discussion}

Elucidation and understanding of the transmission mode, the source and the routes of transmission, of $M$. leprae are essential in developing drastic measures to prevent an infection. Previous sero-epidemiological studies indicated widespread M. leprae infections within a population $(9,10$, 11, 12), and studies by PCR on the distribution of the bacilli also found that many individuals in areas in which leprosy is endemic carried M. leprae on the surface of their nasal cavities $(6,12,13)$. These studies suggested the presence of an infectious source other than that of a patient within the same dwelling. The aim of this study was to clarify

Table 1. TTC genotypes of $M$. leprae detected from the skin and surfaces of nasal mucosa of patients and surfaces of nasal mucosa residents living in the same house.

\begin{tabular}{|c|c|c|c|c|}
\hline $\begin{array}{l}\text { Multi- } \\
\text { family } \\
\text { case }\end{array}$ & $\begin{array}{l}\text { Leprosy patient } \\
\text { (Type of patients) }\end{array}$ & $\begin{array}{c}\text { Contacts } \\
\text { (Relationship) }\end{array}$ & $\begin{array}{c}\text { TTC genotype } \\
\text { (Slit skin) }\end{array}$ & $\begin{array}{l}\text { TTC genotype } \\
\text { (Nasal swabs) }\end{array}$ \\
\hline $\bar{A}$ & MB* & $\begin{array}{l}\text { Wife } \\
\text { Son }\end{array}$ & $\begin{array}{l}11 \\
- \\
-\end{array}$ & $\begin{array}{r}15 \\
14 \\
17\end{array}$ \\
\hline B & $\mathrm{PB}^{* *}$ & Grand mother & $\begin{array}{l}21 \\
-\end{array}$ & $\begin{array}{l}18 \\
15\end{array}$ \\
\hline $\mathrm{C}$ & MB* & $\begin{array}{l}\text { Son } \\
\text { Son }\end{array}$ & $\begin{array}{l}16 \\
- \\
-\end{array}$ & $\begin{array}{l}15 \\
16 \\
15\end{array}$ \\
\hline $\mathrm{D}$ & $\mathrm{MB}^{*}$ & $\begin{array}{l}\text { Daughter } \\
\text { Daughter } \\
\text { Son }\end{array}$ & $\begin{array}{c}13 \\
- \\
- \\
-\end{array}$ & $\begin{array}{c}16 \\
13 \\
16 \\
9\end{array}$ \\
\hline
\end{tabular}

* MB; Multibacilary

**PB; Paucibacillary 
microbiologically whether or not MB cases in the same dwelling represent the main source of infection. Establishing a methodology to discriminate the isolates of $M$. leprae is fundamental for these purposes. Although no useful genotyping methods for epidemiological analysis have been available until in 2000, two genomic divergence of $M$. leprae successfully found based on variable number tandem repeats (VNTRs) $(5,14)$. One of the authors (M. Matsuoka) discovered that $M$. leprae isolates could be divided into two subtypes on the basis of the polymorphism in the rpoT gene. The geograph-

Table 2. Frequency of each genotype observed in patients and household contacts.

\begin{tabular}{c|c|c|c}
\hline \multirow{2}{*}{$\begin{array}{c}\text { No. of } \\
\text { repeats }\end{array}$} & \multicolumn{3}{|c}{ Genotype frequency } \\
\cline { 2 - 4 } & Patients lesion & Nasal mucus & Total \\
\hline 9 & 2 & 1 & $3(3.1 \%)$ \\
11 & 2 & 1 & $3(3.1 \%)$ \\
12 & 6 & 4 & $10(10.3 \%)$ \\
13 & 6 & 6 & $12(12.3 \%)$ \\
14 & 4 & 9 & $13(13.4 \%)$ \\
15 & 4 & 8 & $12(12.3 \%)$ \\
16 & 11 & 12 & $23(23.7 \%)$ \\
17 & 2 & 4 & $6(6.2 \%)$ \\
21 & 2 & 3 & $5(5.2 \%)$ \\
22 & 6 & 4 & $10(10.3 \%)$ \\
\hline Total & 45 & 52 & 97 \\
\hline
\end{tabular}

Table 3. TTC genotypes of $M$. leprae obtained from multi-family cases.

\begin{tabular}{c|c|c}
\hline Case & $\begin{array}{c}\text { TTC genotype of the } \\
\text { bacilli from } \\
\text { supposed index case }\end{array}$ & $\begin{array}{c}\text { TTC genotype of patient } \\
\text { bacilli from patient } \\
\text { secondary case }\end{array}$ \\
\hline 1 & Father: 13 copies & Son:9 copies \\
\hline 2 & Father:22 copies & Son:17copies \\
\hline 3 & Mother: 11copies & Daughter:14 copies \\
\hline 4 & Older brother:16copies & Younger brother: 15 copies \\
\hline
\end{tabular}

ical distribution of each genotype in the world was biased and seemed to be related to prehistoric movement of the human race (14). Nevertheless, the genomic diversity of the rpoT cannot be used for epidemiological tracing of the transmission of leprosy bacilli. Genotyping by Single Nucleotide Polymorphisms (SNPs) is applicable to analyze movement of the human race but neither useful for analyzing transmission. (15). Genotyping to compare diversity of short-tandem-repeat loci on the basis of PCR is feasible for community based molecular epidemiological analysis, since $M$. leprae is not cultivable and shows very low levels of diversion in genomic DNA (16). Variety in the copy numbers of TTC repeats can be used to classify $M$. leprae into a considerable number of subtypes and discriminate isolates for each leprosy case. It is reasonable to assume that if the index case in the same dwelling is the source of infection, the genotypes detected in the house should be identical among the household members. In this study, various types of TTC genotypes were detected from nasal mucosa of the healthy HC.

Results obtained clearly demonstrated that there were families with different TTC genotypes of $M$. leprae on the surface of nasal cavities among the residents in the same dwelling. Therefore, the results of the investigation indicate that these residents are contaminated by bacilli with different genotypes. No variations in genotype among the isolates obtained from various lesions in the same patient were shown. This result consequently enables comparisons of the genotypes of bacilli obtained from different patients. We had identified the existence of TTC genotypes of $M$. leprae that differed between the newly detected family contacts and the supposed index case patient. These results strongly suggest that the bacilli did not originate from a single patient in the dwelling and also indicate the exposure of the family members 
to infectious sources out of the dwelling. Previous sero-epidemiological studies suggested that for the majority of cases, the possible source of infection might be in the environment rather than in direct contact with leprosy patients $(9,14,17)$. The findings by PCR, which revealed the wide distribution of the bacilli among the residents in endemic areas, also indicated that the transmission of the bacilli was not only from the leprosy patients $(6,12,13$, 17, 18). The present study strongly supports these assumptions respecting the infectious source(s). Although many epidemiological observations indicated that the household contact was the risk factor for the development of leprosy $(18,19)$, on the other hand, many new cases occurred among populate without any known contact with patients (20). Therefore the source of the secondary case is not only from his/her household. The tendency seen of the accumulation of patients in some families might be attributed to other conditions such as susceptibility to leprosy infection, which is related to genetic predisposition as well as to acquired factors (21). Two groups of the household leprosy cases showed apparently different TTC genotypes between a father and his son, mother and daughter and among brothers. The inconsistency of the genotypes between $M$. leprae isolates obtained from household cases of patients living in the same dwelling clearly indicates that these patients are not always the source in infections of the other family members.

Though the members of the other groups of leprosy cases other than 4 cases shown in table 3 showed the same genotype, whether those people were truly infected by the patient in the house was unclear. The presence of the same genotype in two cases doesn't necessarily imply the infection was occurred from a patient to family contacts, for some TTC genotypes such as 16 copies were widely distributed in the areas. Other polymor- phisms which can discriminate within a given TTC genotype are needed to elucidate this problem. Better epidemiological analysis could be done by the combination of various genotyping techniques. However, TTC genotyping enabled the subtyping of $M$. leprae into more types than rpoT or SNPs genotyping. Other short polymorphic-tandem-repeat loci exist in $M$. leprae genome $(2,22)$ combination with genotyping using other polymorphisms might be a useful tool for precise epidemiological analysis especially for the strains with same TTC copy numbers. Other genotyping measures depending on other short polymorphic tandem repeat loci are proposed (3).

The frequency of 24 or 25 TTC repeats was the highest in the previous study, which examined $M$. leprae isolates obtained in Cebu, Philippines (5). Bacilli with 10 copies of TTC repeats were most frequently isolated in the present study, and the bacilli with large numbers (such as 37) of TTC repeats were not detected (Table 3 ). It is of interest to compare the frequencies of each genotype in different areas, since the results of a previous study indicated that the spread of the bacilli with specific genotypes was consistent with migration of some human groups $(14,23)$. The evidence resulting from the present molecular epidemiological study indicated the existence of an infectious source other than patients in the same dwelling. Wide distribution of the bacilli among residents $(6,8,12)$ and a high positive ratio of anti-PGL-1 antibody among healthy residents $(10,11)$ suggested that the bacilli existed in certain sources to which people were commonly exposed. Genotyping study of the bacilli obtained at the areas with high leprosy prevalence also suggested infection other than patients $(17,18)$ Taking these results into consideration, the environment seems to be the most likely infectious source as suggested previously (24). However, it has not been elucidated so far. 


\section{Acknowledgement}

We would like to thank Board of Directors, Department of Medical Research, (Lower Myanmar) for their advice and encouragements to our research. We are obliged to Dr. Masako Namisato and Dr. Yoshiko Kashiwabara, Tokyo for their support and encouragement.

\section{References}

1) World Health Organization. Leprosy global situation. Wkly. Epidemiol. Rec. 86: 389-400, 2011

2) Matsuoka M. Recent advances in the molecular epidemiology of leprosy. Jpn J Lepr 78: 6773, 2009

3) Gillis T, Vissa V, Matsuoka M, Young S, Richardus $\mathrm{JH}$, Truman R, Hall B, Brennan P, The IDEAL CONSORTIUM Partners: Characterization of Short tandem repeats for genotyping Mycobacterium leprae. Lepr Rev 80: 250-260, 2009

4) Monot M, Honoré N, Garnier T, Zidane N, Sherafi D, Paniz-Mondolfi A, Matsuoka M, Taylor GM, Donoghue HD, Bouwman A, Mays S, Watson C. Lockwood D, Khamispour A, Dowlati Y, Jianping S, Rea TH, Vera-Cabrera L, Stefani MM., Banu S, Macdonald M, Sapkota BR, Spencer J, Thomas J, Harshman K, Singh P, Busso P, Gattiker A, Rougemont J, Brennan PJ and Cole ST: Comparative genomic and phylogeographic analysis of $\mathrm{Myco}$ bacterium leprae. Nature Genetics 41: 12821289, 2009

5) Shin YC, Lee H, Lee H, Walsh GP, Kim JD, and Cho SN: Variable numbers of TTC repeats in Mycobacterium leprae DNA from leprosy patients and use in strain differentiation. J Clin
Microbiol 38: 4535-4538, 2000

6) Saeki K, Budiawan T, Matsuoka M, Izumi S: Epidemiological significance of $M$. leprae in the residential environment: detection of Mycobacterium leprae on the surface of nasal cavity of inhabitants in a leprosy endemic area using the polymerase chain reaction. Jpn J Dermatol 110: 153-160, 2000 (In Japanese.)

7) Matsuoka M, Zhang L, Budiawan T, Saeki K, Izumi S: Genotyping of Mycobacterium leprae on the basis of polymorphism of TTC repeats for analysis of leprosy transmission. J Clin Microbiol 42: 741-745, 2004

8) Klatser PR, van Beers SM, Madjid B, Day R, de Wit MYL: Detection of Mycobacterium leprae nasal carriers in populations for which leprosy is endemic. J Clin Microbiol 31: 29472951, 1993

9) Abe M, Ozawa T, Minagawa F, Yoshino Y: Immunoepidemiological studies on subclinical infection in leprosy. II. Geographical distribution of seropositive responders with special reference to their possible source of infection. Jpn J Lepr 59: 162-168, 1990

10) Cho SN, Kim SH, Cellona RV, Chan GP, Fajardo TT, Walsh GP, Kim JD: Prevalence of IgM antibodies to phenolic glycolipid I among household contacts and controls in Korea and the Philippines. Lepr Rev 63: 12-20, 1992.

11) Izumi $S$, Budiawan $T$, Saeki K, Matsuoka $M$, Kawatsu K: An epidemiological study on $\mathrm{My}$ cobacterium leprae infection and prevalence of leprosy in endemic villages by molecular biological technique. Indian J Lepr 71: 37-43, 1999.

12) van Beers SM, Izumi S, Madjid B, Maeda $Y$, Day R, Klatser PR: An epidemiological study of leprosy infection by serology and polymerase chain reaction. Int J Lepr Other Mycobact Dis 62: 1-9, 1994 
13) Klatser PR, van Beers SM, Madjid B, Day R, de Wit MYL: Detection of Mycobacterium leprae nasal carriers in populations for which leprosy is endemic. J Clin Microbiol 31: 29472951, 1993

14) Matsuoka $M$, Maeda $S$, Kai $M$, Nakata $N$, Chae GT, Gillis TP, Kobayashi K, Izumi S, Kashiwabara Y. Mycobacterium leprae typing by genomic diversity and global distribution of genotypes. Int J Lepr Other Mycobact Dis 68: 121-128, 2000

15) Monot M, Honoré N, Garnier T, Zidane N, Sherafi D, Paniz-Mondolfi A, Matsuoka M, Taylor GM, Donoghue HD, Bouwman A, Mays S, Watson C. Lockwood D, Khamispour A, Dowlati Y, Jianping S, Rea TH, VeraCabrera L, Stefani MM, Banu S, Macdonald M, Sapkota BR, Spencer J, Thomas J, Harshman K, Singh P, Busso P, Gattiker A, Rougemont J, Brennan PJ, Cole ST: Comparative genomic and phylogeographic analysis of Mycobacterium leprae. Nature Genetics 41: 1282-1289, 2009

16) Douglas Y: Proposal for molecular epidemiology of leprosy. Lepr Rev 74: 11-17, 2003.

17) Cho SN, Kim SH, R. Cellona V, Chan GP, Fajardo TT, Walsh GP, Kim JD: Prevalence of IgM antibodies to phenolic glycolipid I among household contacts and controls in Korea and the Philippines. Lepr Rev 63: 12-20, 1992.

18) Trankar RP, Lavania M, Singh M, SivaSai KRSDR, Jadhav RS: Dynamics of Mycobac- terium leprae transmission in environmental context: Deciphering the role of the environment as a potential reservoir. Infec Genet Evol 12:121-126, 2012

19) Noordeen SK: The epidemiology of leprosy, pp. 29-45. In R. C. Hastings (ed.) Leprosy. Churchill Livingstone, New York, N.Y. 1994

20) Fine PE, Sterne JM, Ponnighaus JM, Bliss L, Saui J, Chihana A, Munthali M, Warandorff DK: Household and dwelling contacts as risk factors for leprosy in northern Malawi. Am J Epidemiol 146: 91-102, 1997

21) de Vries RRP, Ottenhoff TH: Immunogenetics of leprosy, pp. 113-121. In R. C. Hastings (ed.), Leprosy. Churchill Livingstone, New York, N.Y. 1994

22) Zhang L, Budiawan T, Matsuoka M: Diversity of potential short tandem repeats in Mycobacterium leprae and application for Molecular typing. J Clin Microbiol 43: 5221-5229, 2005

23) Matsuoka M, Lopez R, Budiawan T, Kyaw K, Chae GT: Genotypic analysis of Mycobacterium leprae isolates from Japan and other Asian countries reveals global transmission pattern of leprosy. FEMS Microbiol Letter 261: 150154, 2006

24) Kerr-Pontes LRS, Barreto ML, Evagelista CMN, Rodrigues LC, Heukelbach J, Feldmeier H: Socioeconomic, environmental, and behavioral risk factors for leprosy in Northeast Barzil: results of a case-control study. Int J Epidemiol 35: 994-1000, 2006 